\title{
True quantum face of the "exponential" decay law
}

\author{
Krzysztof Urbanowski ${ }^{\mathrm{a}}$ \\ University of Zielona Góra, Institute of Physics, ul. Prof. Z. Szafrana 4a, 65-516 Zielona Góra, Poland
}

Received 25 October 2016 / Received in final form 20 February 2017

Published online 23 May 2017

(C) The Author(s) 2017. This article is published with open access at Springerlink.com

\begin{abstract}
Results of theoretical studies of the quantum unstable systems caused that there are rather widespread belief that a universal feature of the quantum decay process is the presence of three time regimes of the decay process: the early time (initial) leading to the Quantum Zeno (or Anti Zeno) Effects, "exponential" (or "canonical") described by the decay law of the exponential form, and late time characterized by the decay law having inverse-power law form. Based on the fundamental principles of the quantum theory we give the proof that there is no time interval in which the survival probability (decay law) could be a decreasing function of time of the purely exponential form but even at the "exponential" regime the decay curve is oscillatory modulated with a smaller or a large amplitude of oscillations depending on parameters of the model considered.
\end{abstract}

\section{Introduction}

The discovery of radioactivity in the nineteenth century initialized the study of the process of decay of radioactive elements. Experiments have shown that the radioactive decay of the sample of radioactive elements is a process extended in time, and that with the passage of time the number of elements in the sample, which emits radioactive radiation, decreases. These observations and assumption that the decay rate follows the laws of probability led Rutherford and Sody to the formulation of radioactive decay law as a function of time $[1,2]$. This radioactive decay law allows to determine the number $N(t)$ of atoms of the radioactive element at the instant $t$ knowing the initial number $N_{0}=N(0)$ of them at initial instant of time $t_{0}=0$ and has the exponential form: $N(t)=N_{0} \exp [-\lambda t]$, where $\lambda>0$ is a constant. Since then, the belief that the decay law has the exponential form has become common. The rise of Quantum Mechanics led to an understanding that the radioactive decay similarly to the process of emission of photons by excited atoms are time dependent quantum processes. So the question arose how to describe such processes within the quantum theory. Probably the most known attempt to solve this problem is the WeisskopfWigner theory of spontaneous emission $[3,4]$. Considering the excited atomic levels and applying the Shrödinger equation to describe the time evolution Weisskopf and Wigner found that to a good approximation the non-decay probability of the excited levels is a decreasing function of time having exponential form. Further studies of the quantum decay processes showed that basic principles of the quantum theory does not allow them to be described by

${ }^{a}$ e-mail: K.Urbanowski@if.uz.zgora.pl an exponential decay law at very late times [5,6] and at initial stage of the decay process (see e.g. [6] and references therein). Theoretical analysis shows that at late times the survival probability (i.e. the decay law) should tends to zero as $t \rightarrow \infty$ much more slowly than any exponential function of time and that as a function of time it has the inverse power-like form at this regime of time $[5,6]$. There was many unsuccessful attempts to verify experimentally predicted deviations from the exponential form of the decay law at late times regime (see e.g. [7]). The first experimental evidence of deviations of the decay law from exponential form at such a time regime was reported in [8]. The early times properties of the decay process lead to the so called Quantum Zeno Effect $[9,10]$, that is to slowing down sufficiently frequently observed decay process up to stop it down in the case of the continuously observed the unstable system. The experimental confirmation of this effect was reported, e.g. in [11] and recently in [12]. All these results of theoretical and experimental researches caused that there are rather widespread belief that a universal feature of the quantum decay process is the presence of three time regimes of such a decay process: the early time (initial), exponential (or "canonical"), and late time having inverse-power law form [13]. This belief is reinforced by a numerous presentations in the literature of decay curves obtained for quantum models of unstable systems. The typical form of such a decay curve one can find in Figure 1. In this context, each experimental evidence of oscillating decay curve at times of the order of the life time is considered as an anomaly caused by new quantum effects or new interactions (see e.g. [14,15]). The question arises, if indeed in the case of one component quantum unstable systems these oscillations of the decay process 


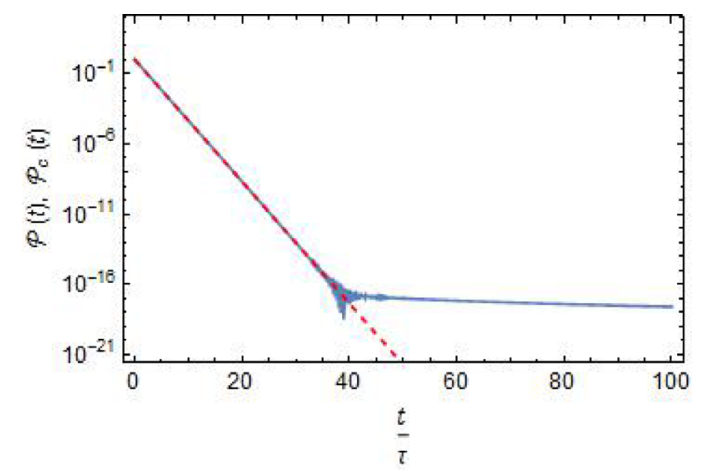

Fig. 1. Decay curves obtained for $\omega_{B W}(E)$ given by equation (5). The case $s_{R}=1000 . \tau=\frac{\hbar}{\Gamma_{0}}$ is the lifetime, $t$ is the time. Solid line: the decay curve $\mathcal{P}(t)=|a(t)|^{2}$. Dotted line: the canonical decay curve $\mathcal{P}_{c}(t)=\left|a_{c}(t)\right|^{2}$.

at the "exponential" regime are an anomaly, or perhaps universal feature of quantum decay processes. Here we give the proof that there is no time interval in which the survival probability (decay law) could be a decreasing function of time of the purely exponential form. We also show that even in the case of a single component unstable system the decay curve has an oscillatory form with a smaller or a large amplitude of oscillations depending on the model considered.

\section{Preliminaries}

The main information about properties of quantum unstable systems is contained in their decay law, that is in their survival probability. Let the reference frame $\mathcal{O}$ be the common inertial rest frame for the observer and for the unstable system. Then if one knows that the system in the rest frame is in the initial unstable state $|\phi\rangle \in \mathcal{H}$, $(\mathcal{H}$ is the Hilbert space of states of the considered system), which was prepared at the initial instant $t_{0}=0$, then one can calculate its survival probability (the decay law), $\mathcal{P}(t)$, of the unstable state $|\phi\rangle$ decaying in vacuum, which equals

$$
\mathcal{P}(t)=|a(t)|^{2},
$$

where $a(t)$ is the probability amplitude of finding the system at the time $t$ in the initial unstable state $|\phi\rangle$,

$$
a(t)=\langle\phi \mid \phi(t)\rangle
$$

and $|\phi(t)\rangle$ is the solution of the Schrödinger equation for the initial condition $|\phi(0)\rangle=|\phi\rangle$ :

$$
i \hbar \frac{\partial}{\partial t}|\phi(t)\rangle=H|\phi(t)\rangle
$$

Here $|\phi\rangle,|\phi(t)\rangle \in \mathcal{H}$, and $H$ denotes the total self-adjoint Hamiltonian for the system considered. Note that if $|\phi\rangle$ represents an unstable state then it cannot be an eigenvector for $H$ : in such a case the eigenvalue equation $H|\phi\rangle=\epsilon_{\phi}|\phi\rangle$ has no solutions for $|\phi\rangle$ under considerations.
There is $|\phi(t)\rangle=U(t)|\phi\rangle$, where $U(t)$ is unitary evolution operator and $U(0)=\mathbb{I}$ is the unit operator. Thus $a(t) \equiv\langle\phi|U(t)| \phi\rangle$. The one-parameter family of unitary operators $U(t)$ forms group: $U\left(t_{1}\right) U\left(t_{2}\right)=U\left(t_{1}+t_{2}\right)$. The the total Hamiltonian $H$ of the system is a generator of this group. This means that operators $H$ and $U(t)$ have common eigenfunctions.

From the results of theoretical studies of the problem which one can find in the literature it is known that the amplitude $a(t)$, and thus the decay law $\mathcal{P}(t)$ of the unstable state $|\phi\rangle$, are completely determined by the density of the energy distribution $\omega(E)$ for the system in this state $[16,17]$,

$$
a(t)=\int_{\text {Spec. }(H)} \omega(E) e^{-\frac{i}{\hbar} E t} d E,
$$

where $\omega(E) \geq 0$ and $a(0)=1$ (see also: $[5,6,18,19]$ ). From this relation and from the Riemann-Lebesgue lemma it follows that $|a(t)| \rightarrow 0$ as $t \rightarrow \infty$. It is because from the normalization condition $a(0)=1$ it follows that $\omega(E)$ is an absolutely integrable function. These properties are the essence of the so-called Fock-Krylov theory of unstable states $[16,17]$ (note that this approach is also applicable in Quantum Field Theory models [20,21]).

Khalfin in [5] assuming that the spectrum, Spec. $(H)=$ $\sigma(H)$, of $H$ must be bounded from below, $(\sigma(H)=$ $\left[E_{\min }, \infty\right)$ and $\left.E_{\min }>-\infty\right)$, that is that $\omega(E)=0$ for $E<E_{\text {min }}$, and using the Paley-Wiener Theorem [22] proved that in the case of unstable states there must be $|a(t)| \geq A \exp \left[-b t^{q}\right]$, for $|t| \rightarrow \infty$. Here $A>0, b>0$ and $0<q<1$. Therefore the decay law $\mathcal{P}(t)$ of unstable states decaying in the vacuum, (1), can not be described by an exponential function of time $t$ if time $t$ is suitably long, $t \rightarrow \infty$, and that for these lengths of time $\mathcal{P}(t)$ tends to zero as $t \rightarrow \infty$ more slowly than any exponential function of $t$. As it was mentioned, this effect was confirmed in experiment described in the Rothe paper [8].

\section{The Breit-Wigner model}

In general the spectral density $\omega(E)$ has properties similar to the scattering amplitude, i.e., it can be decomposed into a threshold factor, a pole-function $P(E)$ with a simple pole (often modeled by a Breit-Wigner) and a smooth form factor $F(E)$ : there is $\omega(E)=\Theta\left(E-E_{\min }\right)(E-$ $\left.E_{\text {min }}\right)^{\alpha_{l}} P(E) F(E)$, where $\alpha_{l}$ depends on the angular momentum $l$ through $\alpha_{l}=\alpha+l$, [6] (see Eq. (6.1) in [6]), $0 \leq$ $\alpha<1$ ) and $\Theta(E)$ is a step function: $\Theta(E)=0$ for $E \leq 0$ and $\Theta(E)=1$ for $E>0$. The simplest choice is to take $\alpha=0, l=0, F(E)=1$ and to assume that $P(E)$ has a Breit-Wigner form. It turns out that the decay curves obtained in this simplest case are very similar in form to the curves calculated for the above described more general $\omega(E)$ (see [18] and analysis in [6]). So to find the most typical properties of the decay curve it is sufficient to make the relevant calculations for $\omega(E)$ modeled by the the Breit-Wigner distribution of the energy density. 
The typical form of the survival probability $\mathcal{P}(t)$ obtained in such a way is presented in Figure 1. The calculations were made for $\omega(E)$ having the Breit-Wigner form $\omega(E) \equiv \omega_{B W}(E)$,

$$
\omega_{B W}(E)=\frac{N}{2 \pi} \Theta\left(E-E_{m i n}\right) \frac{\Gamma_{0}}{\left(E-E_{0}\right)^{2}+\left(\frac{\Gamma_{0}}{2}\right)^{2}},
$$

where $N$ is a normalization constant. The results depend on the parameters of the model $E_{R}=E_{0}-E_{\min }$ and $\Gamma_{0}$ : strictly speaking on the ratio $s_{R} \stackrel{\text { def }}{=} \frac{E_{R}}{\Gamma_{0}}$.

The case $\omega(E)=\omega_{B W}(E)$ is the typical case considered in numerous papers and used therein to model decay processes: among others, the Breit-Wigner model is often used to justify a belief that there exists the "exponential time regime" of the decay process (see e.g. [13,23] and Fig. 1). Therefore it is very important to analyze real form of the decay curves obtained using $\omega(E)=\omega_{B W}(E)$ and this is why we consider this case in this paper. What is more, substituting $\omega_{B W}(E)$ into (4) allows one to find the analytical formula for the amplitude $a(t)$. The result is (see, e.g. [23-25])

$$
\begin{aligned}
a(t)= & N e^{-\frac{i}{\hbar}\left(E_{0}-i \frac{\Gamma_{0}}{2}\right) t} \\
& \times\left\{1-\frac{i}{2 \pi}\left[e^{\frac{\Gamma_{0} t}{\hbar}} E_{1}\left(-\frac{i}{\hbar}\left(E_{R}+\frac{i}{2} \Gamma_{0}\right) t\right)\right.\right. \\
& \left.\left.+(-1) E_{1}\left(-\frac{i}{\hbar}\left(E_{R}-\frac{i}{2} \Gamma_{0}\right) t\right)\right]\right\}
\end{aligned}
$$

where $E_{1}(x)$ denotes the integral-exponential function defined according to [26].

The standard canonical form of the survival amplitude $a_{c}(t)$, is given by the following relation,

$$
a_{c}(t)=\exp \left[-i \frac{t}{\hbar}\left(E_{0}-\frac{i}{2} \Gamma_{0}\right)\right]
$$

$\Gamma_{0}$ is the decay rate and $\frac{\hbar}{\Gamma_{0}}=\tau$ is the lifetime (time $t$ and $\Gamma_{0}$ are measured in the rest reference frame of the particle).

It is convenient to consider the following function

$$
\zeta(t) \stackrel{\text { def }}{=} \frac{a(t)}{a_{c}(t)} .
$$

The reason is simple: there is $|\zeta(t)|^{2}=\mathcal{P}(t) / \mathcal{P}_{c}(t)$, where $\mathcal{P}_{c}(t)=\left|a_{c}(t)\right|^{2}$ is the canonical exponential form of the decay law. Analysis of properties of this function allows one to visualize all the more subtle differences between $\mathcal{P}(t)$ and $\mathcal{P}_{c}(t)$. For example, if one finds a time interval $\left[t_{1}, t_{2}\right]$ such that $\zeta(t)=$ const. for $t \in\left[t_{1}, t_{2}\right]$ this will mean that the survival probability $\mathcal{P}(t)$ has purely exponential form in this time interval.
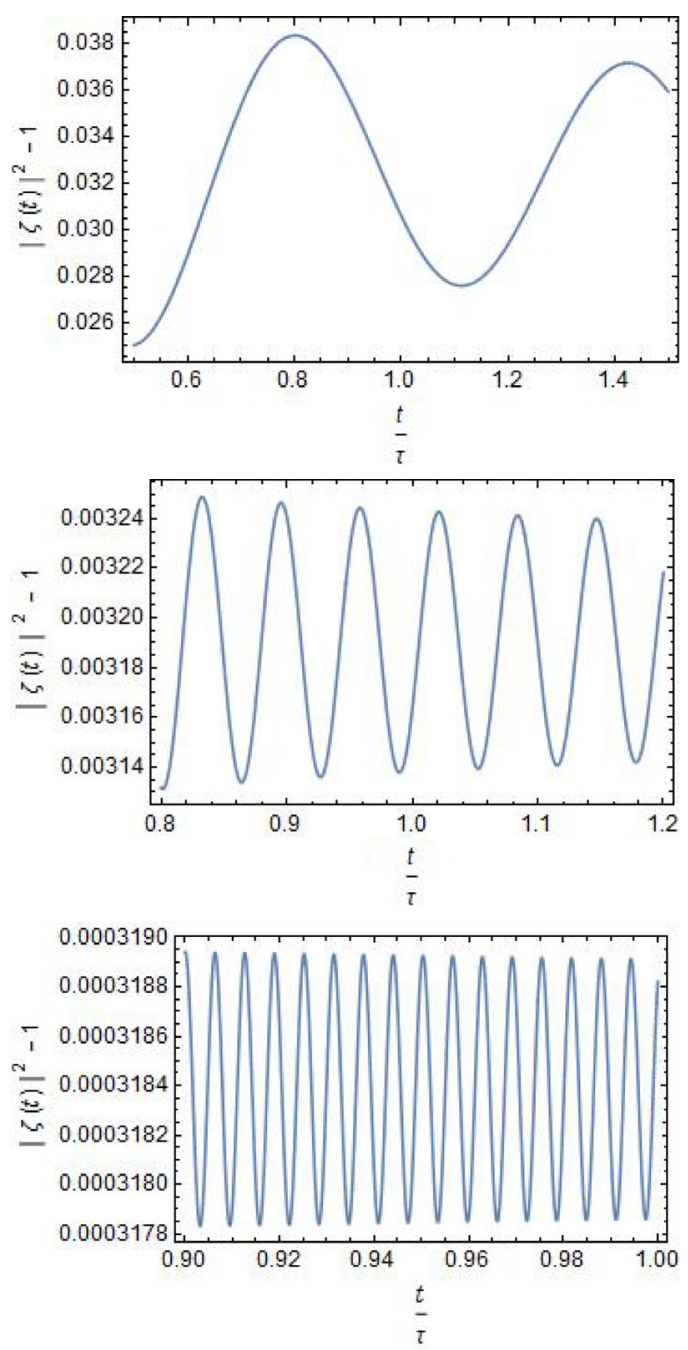

Fig. 2. A comparison of decay curves obtained for $\omega_{B W}(E)$ given by equation (5) with canonical decay curves. Here $\tau$ denotes the lifetime, $t$ is time, $\left(|\zeta(t)|^{2}-1\right) \equiv \frac{\mathcal{P}(t)-\mathcal{P}_{c}(t)}{\mathcal{P}_{c}(t)}$. The top panel: $s_{R}=10$. The middle panel: $s_{R}=100$. The lower panel: $s_{R}=1000$.

The function $\zeta(t)$ takes the following form in the case of the unstable system modeled by $\omega_{B W}(E)$ :

$$
\begin{aligned}
\zeta(t) \equiv & N\left\{1-\frac{i}{2 \pi}\left[e^{\frac{\Gamma_{0} t}{\hbar}} E_{1}\left(-\frac{i}{\hbar}\left(E_{R}+\frac{i}{2} \Gamma_{0}\right) t\right)\right.\right. \\
& \left.\left.+(-1) E_{1}\left(-\frac{i}{\hbar}\left(E_{R}-\frac{i}{2} \Gamma_{0}\right) t\right)\right]\right\} .
\end{aligned}
$$

This function was used to find numerically $|\zeta(t)|^{2}$ for $\omega(E)=\omega_{B W}(E)$. Results of numerical calculations are presented in Figures 2 and 3.

The derivative of $\zeta(t)$ given by (9) equals $[24,25]$

$$
\frac{\partial \zeta(t)}{\partial t}=i \frac{N}{2 \pi} \frac{\Gamma_{0}}{\hbar} e^{\frac{\Gamma_{0}}{\hbar} t} E_{1}\left(-\frac{i}{\hbar}\left(E_{R}+\frac{i}{2} \Gamma_{0}\right) t\right) .
$$

From the properties of the integral-exponential function $E_{1}(x)$ it follows that the equation $\frac{\partial \zeta(t)}{\partial t}=0$ 

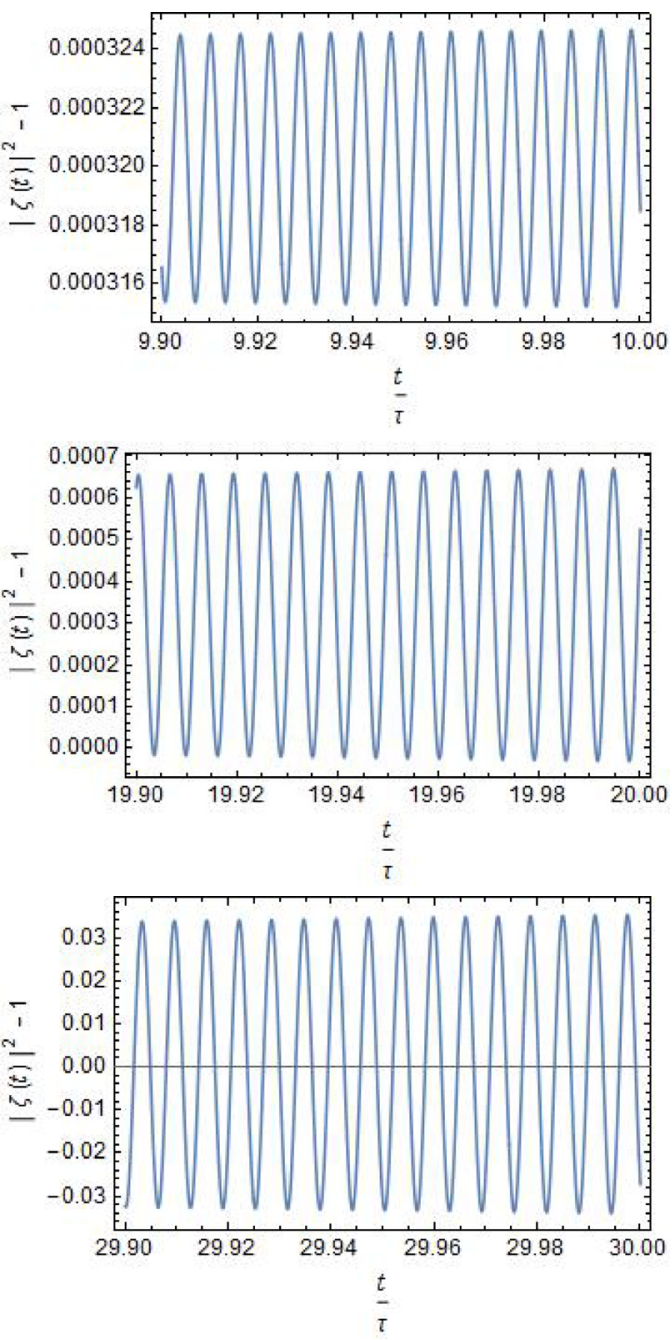

Fig. 3. A comparison of decay curves obtained for $\omega_{B W}(E)$ given by equation (5) with canonical decay curves. The case $s_{R}=1000$. Here $\tau$ is the lifetime, $t$ is time, $\left(|\zeta(t)|^{2}-1\right)=$ $\frac{\mathcal{P}(t)-\mathcal{P}_{c}(t)}{\mathcal{P}_{c}(t)}$. The top panel: $t / \tau \in[9.90,10.00]$, the middle panel: $t / \tau \in[19.90,20.00]$, the lower panel: $t / \tau \in[29.90,30.00]$.

can be satisfied at most for some isolated values of time $t$. So, from the formula (10) the conclusion follows: within the model considered there is no time interval $\left[t_{1}, t_{2}\right]$ (where $t_{1}<t_{2}$ ), in which $\zeta(t)=$ const. for $t \in\left[t_{1}, t_{2}\right]$, that is, there is no time interval in which the survival probability $\mathcal{P}(t)$ has a pure exponential form. This conclusion explains the results presented in Figures 2 and 3.

\section{The general case}

Results obtained for $\omega(E)=\omega_{B W}(E)$ and presented in Figures 2 and 3 can be understood as the interference of the pole contribution, $a_{\text {pole }}(t)$, into the survival probability $a(t)$ and the cut contribution, $a_{\text {cut }}(t)$, to $a(t) \equiv$ $a_{\text {pole }}(t)+a_{\text {cut }}(t)$. Analogous effects take place in all models of unstable states, in which unstable states are defined by poles of $\omega(E)$ in the complex plane. Within the much more general Fock-Krylov theory [16,17] of unstable states the only condition that must be met by $\omega(E)$ is an absolute integrability of $\omega(E)$ : if $\omega(E)$ is an absolutely integrable function (even without the poles in the complex plane) then the Riemann-Lebesque lemma ensures that $\mathcal{P}(t)=|a(t)|^{2} \rightarrow 0$ as $t \rightarrow \infty$, where $a(t)$ is given by (4). The question arises whether, in the most general case of $\omega(E)$ and $a(t)$ defined by (4) (i.e. within the Fock-Krylov theory), the effect described in the previous section takes place or not.

So let us assume that the survival amplitude $a(t)$ is defined by (2) and can be represented as a Fourier transform (4) of some absolutely integrable $\omega(E)$. Now we can consider the general case of $\zeta(t)$ defined using this general $a(t)$. From the definition (8) it follows that the equivalent form of $\zeta(t)$ is

$$
\zeta(t) \equiv e^{+i \frac{t}{\hbar}\left(E_{0}-\frac{i}{2} \Gamma_{0}\right)} a(t)
$$

Hence

$$
\frac{\partial \zeta(t)}{\partial t}=\frac{i}{\hbar}\left(E_{0}-\frac{i}{2} \Gamma_{0}\right) \zeta(t)-\frac{i}{\hbar} h(t) \zeta(t),
$$

where

$$
h(t) \stackrel{\text { def }}{=} i \hbar \frac{1}{a(t)} \frac{\partial a(t)}{\partial t},
$$

is the effective Hamiltonian governing the time evolution in the subspace of unstable states $\mathcal{H}_{\|}=P \mathcal{H}$ and $P=$ $|\phi\rangle\langle\phi|$ (see [27] and also [24,25] and references therein). The subspace $\mathcal{H} \ominus \mathcal{H}_{\|}=Q \mathcal{H}=(\mathbb{I}-P) \mathcal{H}$ is the subspace of decay products. The equivalent formula for $h(t)$ has the following form

$$
h(t) \equiv \frac{\langle\phi|H| \phi(t)\rangle}{a(t)}
$$

If $\langle\phi|H| \phi\rangle$ exists then using unitary evolution operator $U(t)$ and projection operators $P$ and $Q$ the last relation can be rewritten as follows

$$
h(t)=\langle\phi|H| \phi\rangle+\frac{\langle\phi|H Q U(t)| \phi\rangle}{a(t)} .
$$

Let us consider an auxiliary function

$$
\chi_{\omega}(t) \stackrel{\text { def }}{=} \int_{\text {Spec. }(H)} E \omega(E) e^{-\frac{i}{\hbar} E t} d E .
$$

Using $\chi_{\omega}(t)$ one can express the expectation value $\langle\phi|H| \phi\rangle$ of $H$ as follows

$$
\langle\phi|H| \phi\rangle \equiv \chi_{\omega}(0)
$$

So if $\omega(E)$ is the absolutely integrable as required then $\langle\phi|H| \phi\rangle$ exist if $\left|\chi_{\omega}(0)\right|<\infty$ and this is the only condition 
for the density $\omega(E)$ that guarantees the existence of the relation (15) for all $t \geq 0$. Note that this condition is rather strong but the Breit-Wigner model shows that relations (13) and (14) lead to finite and well defined $h(t)$ for $t>0$ even if this strong condition is not satisfied (see e.g., $[24,25,28])$. From (14) one conludes that $h(t)$ can exist for $t>0$ if the following two conditions are satisfied: $\left|\chi_{\omega}(t)\right|<\infty$ for $t>0$ and $\left|\chi_{\omega}(t)\right| \leq \alpha|a(t)|$ for $t \rightarrow \infty$, where $\alpha=$ const. $>0$.

Let us assume now that $\langle\phi|H| \phi\rangle$ exists and there exists instants $0<t_{1}<t_{2}<\infty$ of time $t$ such that for any $t \in\left(t_{1}, t_{2}\right)$ there is $\zeta(t)=\zeta\left(t_{1}\right)=\zeta\left(t_{2}\right)=$ const. $\stackrel{\text { def }}{=}$ $c_{\phi} \neq 0$. In this case there should be $\frac{\partial \zeta(t)}{\partial t}=0$ for all $t \in\left(t_{1}, t_{2}\right)$. Taking into account that by definition $\zeta(t) \neq 0$ from (12) we conclude that it is possible only and only if for $t_{1} \leq t \leq t_{2}$,

$$
h(t)-\left(E_{0}-\frac{i}{2} \Gamma_{0}\right)=0,
$$

that is if and only if

$$
h(t)=h\left(t_{1}\right)=h\left(t_{2}\right)=\text { const. } \stackrel{\text { def }}{=} c_{h} \neq 0,
$$

for $t_{1} \leq t \leq t_{2}$. Using (15) and the property $|\phi(t)\rangle=$ $U(t)|\phi\rangle$ one concludes that the equality $h(t)=h\left(t_{2}\right)=c_{h}$ can take place if

$$
\frac{\langle\phi|H Q U(t)| \phi\rangle}{a(t)}=\frac{\left\langle\phi\left|H Q U\left(t_{2}\right)\right| \phi\right\rangle}{a\left(t_{2}\right)} .
$$

Taking into account the group properties of the oneparameter family of unitary operators $U(t)$ we can use in (20) the product $U(t) U\left(t_{2}-t\right) \equiv U\left(t_{2}\right)$ instead of $U\left(t_{2}\right)$. Next using the complex function $\lambda\left(t_{2}, t\right) \stackrel{\text { def }}{=} \frac{a\left(t_{2}\right)}{a(t)}$ one can replace the relation (20) by the following one

$$
\left\langle\phi \left| H Q U(t)\left[\lambda\left(t_{2}, t\right)|\phi\rangle-U\left(t_{2}-t\right)|\phi\rangle\right]=0 .\right.\right.
$$

This condition can be satisfied in two cases: the first one is

$$
U\left(t_{2}-t\right)|\phi\rangle-\lambda\left(t_{2}, t\right)|\phi\rangle=0,
$$

and the second one occurs when

$$
\left[\lambda\left(t_{2}, t\right)-U\left(t_{2}-t\right)\right]|\phi\rangle \neq 0
$$

and vectors $(\langle\phi| H)^{+}=H|\phi\rangle$ and

$$
Q U(t)\left[\lambda\left(t_{2}, t\right)|\phi\rangle-U\left(t_{2}-t\right)|\phi\rangle\right]
$$

are orthogonal to each other.

The first case means that $\frac{\partial \zeta(t)}{\partial t}=0$ if and only if the vector $|\phi\rangle$ representing an unstable state of the system is an eigenvector for the unitary evolution operator $U(t)$. As we noted earlier the evolution operator $U(t)$ and the total Hamiltonian $H$ of the system have common eigenvectors. This means that $\frac{\partial \zeta(t)}{\partial t}=0$ for $t \in\left(t_{1}, t_{2}\right)$ if and only if the unstable state $|\phi\rangle$ of the system is an eigenvector for $H$, which is in contradiction with the property that the vector $|\phi\rangle$ representing the unstable state cannot be the eigenvector for the total Hamiltonian $H$.

The second case: from the definition of the projectors $P$ and $Q$ it follows that this case can be realized only if the vector $H|\phi\rangle$ is proportional to the vector $|\phi\rangle: H|\phi\rangle=\alpha_{\phi}|\phi\rangle$, that is similarly to the first case $\frac{\partial \zeta(t)}{\partial t}=0$ if and only if the vector $|\phi\rangle$ representing the unstable state of the system considered is an eigenvector for the total Hamiltonian $H$, which is again in clear contradiction with the condition that the vector $|\phi\rangle$ representing the unstable state cannot be the eigenvector for the total Hamiltonian $H$.

Taking into account implications of the above two possible realizations of the relation $(21)$ we conclude that the supposition that such time interval $\left[t_{1}, t_{2}\right]$ can exist that $\zeta(t)=$ const. $=\zeta\left(t_{1}\right)=\zeta\left(t_{2}\right)$ for $t \in\left(t_{1}, t_{2}\right)$ is false. So taking into account the definition of $\zeta(t)$ the following conclusion follows: within the approach considered in this paper for any time interval $\left[t_{1}, t_{2}\right]$ the decay law can not be described by the exponential function of time. This conclusion is the general one. It does not depend on models of quantum unstable states and confirms the similar conclusion drawn earlier for the Breit-Wigner model.

\section{Final remarks}

Summing up the oscillating decay curves of one component unstable system can not be considered as something extraordinary or as anomaly: it seems to be a universal feature of the decay process. Oscillatory modulated decay curves are usually observed in two - or more component unstable systems. A typical example of such systems is a neutral meson complex. From the results presented above it follows that such an effect can be also observed in one component quantum unstable systems at the "exponential" decay regimes of times. What is more the oscillatory modulation of decay curves at the "exponential" decays regime takes place even in the quantum unstable system modeled by the Breit-Wigner distribution of the energy density. In general, the oscillatory modulation of the survival probability at the "exponential" decay regime and thus the decay curves with model depending amplitude and oscillations period takes place even in the case of one component unstable systems modeled by any physically acceptable form of $\omega(E)$. This is a true quantum picture of the decay process at the so-called "exponential" regime of times which should be taken into account when interpreting decay experiments with one component unstable systems.

In the light of the foregoing considerations, the question arises is it possible to observe the mentioned phenomenon and how to to this? From results of the model calculations presented in Figures 2 and 3 it follows that at the initial stage of the "exponential" (or "canonical") decay regime the amplitude of oscillations of the survival probability in typical cases seems to be much less than the accuracy of detectors. Then with increasing time the amplitude of oscillations grows (see Fig. 3), which increases the chances of observing them. It seems that the analysis 
of the Rothe experiment [8] can help to prepare such experimental devices that could detect these oscillations even at times of the order of the lifetime or a few lifetimes. Simply, similarly to the Rothe experiment, such molecules must be found that the ratio $s_{R}=\frac{E_{0}-E_{m i n}}{\Gamma_{0}}$ was small for their excited levels. Results presented in Figure 2 reinforce this conclusion: in top panel one sees that for $s_{R}=10$ the amplitude of deviations of the decay law $\mathcal{P}(t)$ from the pure exponential form, $\mathcal{P}_{c}(t)$, which are described by $\left(\left.\zeta(t)\right|^{2}-1\right)=\frac{\mathcal{P}(t)-\mathcal{P}_{c}(t)}{\mathcal{P}_{c}(t)}$, are of the order of $2-3 \%$ at times of the order of the lifetime and period of these oscillations is relatively long. For $s_{R}=100$ these deviations are of the order of 3 per mille (see Fig. 2, the middle panel). Of course the amplitude of these deviations for times of the order a few lifetimes is much larger (see Fig. 3), which enhances chance of observing them. Summing up in order to observe the above discussed oscillations one needs a molecules having such excited levels that the coefficient $s_{r}$ is small for them and also one should have a possibility to register the very large number of events (that is transitions from the excited levels of these molecules to lower stable levels). In addition having such molecules one should also have an apparatus that guarantees the measurement accuracy significantly better than the amplitude of oscillations of the quantity $\frac{\mathcal{P}(t)-\mathcal{P}_{c}(t)}{\mathcal{P}_{c}(t)}$ and time resolution of the detectors should be much better than half period of these oscillations.

\section{Author contribution statement}

I hereby declare that I am the author of the work and all the theoretical results and all results of numerical calculations presented therein are obtained by me.

Open Access This is an open access article distributed under the terms of the Creative Commons Attribution License (http://creativecommons.org/licenses/by/4.0), which permits unrestricted use, distribution, and reproduction in any medium, provided the original work is properly cited.

\section{References}

1. E. Ruthheford, Philos. Mag. XLIX, 1 (1900)

2. E. Rutherford, F. Soddy, Philos. Mag. IV, 370 (1902)

3. V.F. Weisskopf, E.T. Wigner, Zeitschrift für Physik 63, 54 (1930)
4. V.F. Weisskopf, E.T. Wigner, Zeitschrift für Physik 65, 18 (1930)

5. L.A. Khalfin, Zhurnal Eksperimentalnoi i Teoreticheskoi Fiziki (USSR) 33, 1371 (1957) [in Russian], [Soviet Phys. J. Exp. Theor. Phys. 6, 1053 (1958)]

6. L. Fonda, G.C. Ghirardii, A. Rimini, Rep. Prog. Phys. 41, $587(1978)$

7. E.B.Norman, S.B. Gazes, S.G. Crane, D.A. Bennett, Phys. Rev. Lett. 60, 2246 (1988)

8. C. Rothe, S.I. Hintschich, A.P. Monkman, Phys. Rev. Lett. 96, 163601 (2006)

9. B. Misra, E.C.G. Sudarshan, J. Math. Phys. 18, 745 (1977)

10. M.C. Fischer, B. Gutiérrez-Medina, M.G. Raizen, Phys. Rev. Lett. 87, 040402 (2001)

11. W.M. Itano, D.J. Heinzen, J.J. Bollinger, D.J. Wineland, Phys. Rev. A 41, 2295 (1990)

12. Y.S. Patil, S. Chakram, M. Vengalattore, Phys. Rev. Lett. 115, 140402 (2015)

13. M. Peshkin, A. Volya, V. Zelevinsky, Europhys. Lett. 107, 40001 (2014)

14. Yu.A. Litvinov, F. Bosch, N. Winckler, D. Boutin, H.G. Essel, T. Faestermann, H. Geissel, S. Hess, P. Kienle, R. Knöbel, C. Kozhuharov, J. Kurcewicz, L. Maier, K. Beckert, P. Beller, C. Brandau, L. Chen, C. Dimopoulou, B. Fabian, A. Fragner, E. Haettner et al., Phys. Lett. B 664, $162(2008)$

15. P. Kienle, F. Bosch, P. Bühler, T. Faestermann, Yu.A. Litvinov, N. Winckler, M.S. Sanjari, D.B. Shubina, D. Atanasov, H. Geissel, V. Ivanova, X.L. Yan et al., Phys. Lett. B 726, 638 (2013)

16. N.S. Krylov, V.A. Fock, Zhurnal Eksperimentalnoi i Teoreticheskoi Fiziki (USSR) 17, 93 (1947) [in Russian]

17. V.A. Fock, Fundamentals of Quantum Mechanics (Mir Publishers, Moscow, 1978)

18. N.G. Kelkar, M. Nowakowski, J. Phys. A: Math. Theor. 43, 385308 (2010)

19. F. Giraldi, Eur. Phys. J. D 69, 5 (2015)

20. F. Giacosa, Found. Phys. 42, 1262 (2012)

21. M.L. Goldberger, K.M. Watson, Collision theory (Wiley, 1964)

22. R.E.A.C. Paley, Fourier transforms in the complex domain (American Mathematical Society, New York, 1934)

23. K.M. Sluis, E.A. Gislason, Phys. Rev. A 43, 4581 (1991)

24. K. Urbanowski, Eur. Phys. J. C 58, 151 (2008)

25. K. Urbanowski, Open Physics 7, 696 (2009) (Formerly: Central European Journal of Physics)

26. M. Abramowitz, A.I. Stegun, Handbook of Mathematical Functions with Formulas, Graphs, and Mathematical Tables (Dover Publications Inc., New York, 1964)

27. K. Urbanowski, Phys. Rev. A 50, 2847 (1994)

28. K. Urbanowski, K. Raczyńska, Phys. Lett. B 731, 236 (2014) 